\title{
Retos metodológicos en el estudio del sistema de medios informativos en el Perú
}

\author{
Methodological challenges in the study of Peruvian news \\ media system
}

Desafios metodológicos no estudo do sistema de mídia da informação no Peru

Jessica Retis, Universidad de Arizona, Tucson, Estados Unidos (jessicaretis@arizona.edu) Lilian Kanashiro, Universidad de Lima, Lima, Perú (1kanashi@ulima.edu.pe) Wendy Domenack, Universidad de Lima, Lima, Perú (wdomenac@ulima.edu.pe)

\begin{abstract}
RESUMEN|Un sistema de mediosinformativos sólido es crucial para enfrentar diversos tipos de crisis. No obstante, caracterizar dichos sistemas en países latinoamericanos plantea dilemas propios de una institucionalización estructuralmente débil, de procesos de modernización acelerados o en curso. Nuestro objetivo es reconocer los desafíos metodológicos en la constitución de una base de datos de medios informativos que permitan análisis consistentes y comparativos sobre las condiciones del ejercicio periodístico en el mundo. Para ello, nos basamos en el caso peruano, que no cuenta con bases de datos, de medios ni de periodistas de alcance nacional y en el que la institucionalización de los medios es precaria. Hemos utilizado como estrategia metodológica la triangulación de fuentes. Entre las principales dificultades está la informalidad dentro de los medios formales, una huella digital precaria y la insuficiencia de categorizaciones basadas en un sistema analógico en una sociedad en proceso de transmedialización.
\end{abstract}

PALABRAS CLAVE: medios de comunicación; periodismo; metodología; Perú.

Retis, J., Kanashiro, L., \& Domenack, W. (2021). Retos metodológicos en el estudio del sistema de medios informativos en el Perú. Cuadernos.info, (50), 1-21. https://doi.org/10.7764/cdi.50.27321 
ABSTRACT / A strong media system is crucial when facing various types of crises. In Latin America, it becomes challenging to characterize media systems due to the weakness of their structural institutionalization as well as the modernization processes. In this contribution, we address methodological challenges when elaborating a database of news media outlets that could allow us to conduct consistent and comparative analyses ofjournalism practices with an international scope. When analyzing the Peruvian case, we face two main challenges. On the one hand, there is no national census of journalists or news media outlets; on the other hand, the institutionalization of Peruvian media remains precarious. Facing these challenges, we used a triangulation of sources as methodological strategy to elaborate a reliable survey despite the evident informal nature of certain outlets, the precarious digital identification, and the traditional categorizations that were mostly based on legacy media that become insufficient when examining these practices in a transmedia scenario.

KEYWORDS: media; journalism; methodology; Peru.

RESUMo | Um sistema de mídia sólido resulta crucial para lidar com diferentes tipos de crises. No entanto, caracterizar os sistemas em países da América Latina se confronta aos dilemas próprios de uma institucionalização estruturalmente débil ou de processos de modernização acelerados ou em andamento. Nosso objetivo é reconhecer os desafios metodológicos na elaboração de uma base de dados de mídias jornalísticas que permitam análises consistentes e comparativos sobre as condições do exercício jornalístico no mundo. Para isso, fundamentamo-nos no caso peruano que não conta com bases de dados de mídias e nem de jornalistas de alcance nacional; e no qual a institucionalização das mídias é precária. Temos utilizado como estratégia metodológica a triangulação de fontes. Entre as principais dificuldades temos a informalidade dentro dos meios formais, uma impressão digital precária e a insuficiência das categorizações baseadas em um sistema analógico em uma sociedade em processo de transmídialização.

PALAVRAS-CHAVE: mídia; jornalismo; metodologia; Peru. 


\section{INTRODUCCIÓN}

Los sistemas de medios de los países latinoamericanos enfrentan dilemas propios de una institucionalización estructuralmente débil, procesos de modernización acelerados o en curso, lo que dificulta caracterizarlos. Una tendencia encontrada en la región es la alta concentración de propiedad de medios (Sinclair, 1999; Becerra \& Mastrini, 2006, 2009, 2017). Este sistema oligopólico tiende a acaparar los ingresos publicitarios y dominar la producción de flujos de información (Waisbord, 2000; Protzel, 1994, 2014). En Perú se han producido sinergias concéntricas que han derivado en una reforzada presencia de conglomerados de medios informativos (Gargurevich, 2012; Impresos, n.d.; Open Society Foundations, 2010; Lovatón, 2016). Estudiar el sistema de medios informativos en este país presenta una serie de retos metodológicos, debido a la escasez de datos fidedignos que permitan obtener una fotografía clara del entretejido institucional sobre el que se apoyan los flujos de información. Por otro lado, el carácter centralista de la producción informativa desdibuja la importancia de los medios regionales y locales. Como consecuencia, las dinámicas en las otras 23 regiones del país resultan prácticamente invisibles.

Debido a que en Perú no se cuenta con un registro unificado que ofrezca una clara idea de las características del ecosistema mediático en general, este proyecto de investigación busca contribuir a elaborar una cartografía indicativa del estado de los medios informativos. Buscamos identificar cuántos medios informativos operan, los conglomerados predominantes que concentran la propiedad y la distribución geográfica de los flujos informativos en el país. Para sistematizar el ecosistema mediático peruano se enfrenta una serie de retos metodológicos, que puedan constituir un marco referencial para estudios similares en la región. Por ello nos preguntamos ¿Cuáles son los principales retos metodológicos para estudiar el ecosistema mediático peruano?

\section{MARCO TEÓRICO}

Estudiar el sistema de medios a nivel nacional plantea una serie de retos. Mientras que la investigación en medios y comunicación ha experimentado una globalización tanto institucional como disciplinar (Waisbord, 2009; Boczkowski \& Mitchelstein, 2019), los análisis comparados requieren de una mayor claridad conceptual y teórica, puesto que sin una brújula conceptual la investigación comparada corre el riesgo de vararse (Hanitzsch et al., 2019; Gurevitch \& Blumler, 2004; Norris, 2009). Tal como plantean Hanitzsch y sus colegas (2019), la demanda del universalismo teórico puede conducir a una medición insensible con la realidad de cada país y los esfuerzos por adecuar el análisis del nivel local pueden resultar frustrantes al acceder al análisis comparado y sistemático. Por ello, nuestro estudio busca conformar una base teórica que incorpore las especificidades del contexto peruano, pero que también pueda compararse a nivel regional y mundial. 
Por mucho tiempo el modelo hegemónico occidental del periodismo ha conceptualizado al periodista como un profesional independiente y verificador de la información en aras del servicio público (Nerone, 2013), lo que es producto de una ideología ocupacional exportada al mundo como estándar profesional de medición (Golding, 1977). La concentración de estudios en el Norte Global ha producido un sesgo que relaciona al periodismo con la idea de democracia, hecho que ocurre en una minoría de la población mundial (Hanitzsch et al., 2019). Paradójicamente, la producción académica ha experimentado una tendencia antidemocrática que ha privilegiado un mundo periodístico más estrecho (Zelizer, 2013). Waisbord y Mellado (2014) reclamaron la urgente necesidad de desoccidentalizar el conocimiento científico sobre las realidades comunicativas e informativas. El Sur Global requiere hacerse escuchar con mayor contundencia en aras de la democratización de la investigación y, en este sentido, Latinoamérica tiene mucho que aportar (Retis \& Sierra, 2011).

En el marco del Worlds of Journalism se conceptualiza al periodismo como una institución discursiva, más allá de si el periodismo es una profesión, una ocupación, un oficio, un negocio (Zelizer, 2004) o el perro guardián (watchdog) de las instituciones gubernamentales. Al incorporar aproximaciones desoccidentalizadas, se plantea considerar que en otras regiones los periodistas asumen la responsabilidad de preservar la armonía en la sociedad o el respeto a la autoridad (Masterton, 1996; $\mathrm{Xu}, 2005)$ o tienen un rol más activo y participante dentro del discurso político (Hanitzsch et al., 2011; Pintak, 2014). Se plantea, además, incorporar a la discusión las nuevas formas de ejercer la profesión, así como nuevas perspectivas teóricas, adoptando el marco del institucionalismo discursivo, planteamiento que viene desarrollándose desde la ciencia política (Schmidt, 2008, 2010). En este sentido, se proponen dos aproximaciones teóricas centrales: que el periodismo es una institución social y que es discursivamente (re)creado (Hanitzsch \& Vos, 2017; 2018; Hanitzsch et al., 2019). En el caso de Latinoamérica, como afirma Mellado (2009), uno de los problemas que surgen a la hora de estudiar el periodismo y al periodista es lograr definir el marco estructural común que lo(s) define.

En este artículo nos centramos en el carácter institucional para poner en perspectiva internacional la realidad peruana. El marco corporativo del periodismo se compone de reglas, convenciones y prácticas que son propicias, limitantes y constitutivas. En este andamiaje se incluyen estructuras formales, así como reglas y procedimientos informales (Hanitzsch et al., 2019). En el contexto latinoamericano, tal como afirmó Waisbord (2009), los estudios continúan explorando los prospectos del periodismo democrático, pero se vienen incorporando debates sobre la globalización, el cosmopolitanismo y el nuevo institucionalismo. Estudiar las estructuras políticas y económicas continúa siendo muy relevante, debido a que los viejos problemas persisten. En primer lugar, la connivencia entre el aparato estatal 
y los intereses mercantiles continúa vulnerando las posibilidades de los medios democráticos. En segundo lugar, el surgimiento de administraciones populistas y conservadoras en la región en años recientes ha introducido conflictos entre los gobiernos y las organizaciones periodísticas. En tercer lugar, es relevante que las investigaciones avancen en explorar los lazos entre prensa y estatus quo. Asimismo, se han introducido nuevas cuestiones, como los avances tecnológicos, que han supuesto cambios en los modos de reporteo y producción de noticias y han facilitado también el surgimiento de nuevos modelos periodísticos (cívico, independiente, sin fines de lucro, entre otros) (Waisbord \& Mellado, 2014).

Los estudios sobre la prensa en Perú han señalado las tendencias de las últimas décadas (Vivas, 2008; Freundt-Thurne et al., 2013; Cueva et al., 2020). La historia contemporánea del sistema de medios informativos retrata una fuerte concentración de propiedad, una estrecha o tirante relación con el poder político y una tercera vía en la que surgen nuevos modelos del ejercicio profesional. Tal como apunta Protzel (2014), resulta imprescindible incorporar en nuestra reflexión los contextos socioculturales, sociolingüísticos y socioeconómicos en la concepción de las audiencias periodísticas. En su crítica, señala que el término coevolución utilizado para explicar el crecimiento paralelo de los medios masivos con los sistemas políticos no resulta aplicable al caso peruano. La descomposición del tejido social coetáneas con la implantación de medidas neoliberales de orden público reconfiguraron el sistema de medios y la relación con el gobierno. Los beneficios de la prensa popular escondieron una serie de subsidios estatales y los servicios de inteligencia bajo el mando de Fujimori. Sin embargo, luego de su mandato, la lectoría de este tipo de prensa continuaría en aumento, resultando ser un modelo de negocio lucrativo.

Otras investigaciones analizan los riesgos que suponen la tendencia a la concentración de medios en el Perú (Santillán, 2017) particularmente significativa en el caso de la prensa escrita (Acevedo, 2016). También se ha examinado el peligro que la concentración de medios supone para la democracia y la libertad de expresión (Chanduvi, 2014; Acevedo, 2017). Los conglomerados de medios, en el caso peruano, además, han ido avanzando en sus estrategias empresariales hasta convertirse en los que algunos han llamado los dueños de la noticia, para hacer hincapié en el estrecho margen de control de los flujos de información en manos de unos pocos empresarios (Castilla et al., 2016). Por otro lado, el país se está convirtiendo en un territorio de riesgo para el ejercicio profesional, tal como lo han venido denunciando sus representantes (Lainez, 2014).

La relación entre los medios y los presidentes populistas es la responsable de los dos capítulos más importantes en la historia de los medios contemporáneos de Perú (Gargurevich, 2012). El primero es la confiscación de periódicos y estaciones 
de televisión por parte del gobierno militar de Velasco Alvarado en 1974, y el segundo es la extensa corrupción de las líneas editoriales durante el gobierno de Fujimori en los años noventa. Ambos episodios han contribuido a una pérdida general de credibilidad de la prensa. La televisión peruana sigue siendo centralista. La mayoría de sus contenidos se producen en la capital y se transmiten al resto del país a través de estaciones repetidoras (Cueva et al., 2020). La transición a la televisión digital terrestre (TDT) se remonta a noviembre de 2006; sin embargo, la señal digital no se lanzó oficialmente hasta el 30 de marzo de 2010, cuando TVPerú comenzó a transmitir en alta definición. Se espera que la migración al sistema digital termine en 2024 (Freundt-Thurne et al., 2013). El modelo de negocio de la televisión y la radio peruanas se ha desarrollado de manera semejante al modelo liberal occidental basado en la venta de publicidad. La bonanza económica de los años cincuenta favoreció el crecimiento de grupos de radio y, poco más adelante, la consolidación de conglomerados mediáticos. Poco a poco, el panorama fue consolidando la centralización de las estrategias comerciales en la capital, lo que hemos venido comprobando de manera crítica en nuestra revisión de datos reciente.

En su estudio comparado sobre América Latina, Becerra y Mastrini (2006) encontraron que la concentración de la prensa diaria en el Perú alcanzaba un nivel muy alto con relación a la facturación y un nivel más bajo en materia de circulación. En el caso de la radio, hallaron que el nivel de concentración de propiedad era superior al promedio regional. Esto es por la concentración de inversión publicitaria. La concentración de los ingresos es muy relevante si tomamos en cuenta que solo cuatro de mil radios contabilizadas obtienen más de $66 \%$ de los ingresos. Según su baremo, la televisión abierta en Perú alcanza un altísimo nivel de concentración, tanto en facturación como en audiencias. Este se potencia porque concentra más de $75 \%$ de la inversión publicitaria. Además, el dominio del mercado del primer operador de televisión de cable se erguía como el nivel de concentración más alto de todos los países latinoamericanos estudiados por Becerra y Mastrini (2006). En su más reciente estudio regional, que no incluye datos de Perú, se destacan las siguientes sugerencias para el análisis: incorporar los efectos de la profunda transformación tecnológica, las infraestructuras que se usan para producir y distribuir contenidos, las estrategias comerciales, el vínculo con los usuarios y los procesos de trabajo. Sostienen que la concentración viene provocando una reducción de las fuentes informativas, menor pluralidad de emisores, unificación de la línea editorial, empobrecimiento de la deliberación en el espacio público, una relativa homogeneización de los géneros y formatos, predominancia de estilos y temáticas, y una concomitante oclusión de temas y formatos, así como la tendencia a la exclusividad del derecho de distribución/exhibición de eventos de interés general. Todo ello potencia la precarización del empleo y un debilitamiento de la calidad informativa (Becerra \& Mastrini, 2017). 


\section{METOdOLOGÍA}

Hoy en día no existe un registro unificado de medios informativos en el Perú. Por ello, nos planteamos la tarea de elaborar un diseño que conjugara información estatal, pública y privada. El estudio se inició el 2019 y se encuentra en la fase inicial del proceso, por lo que hemos elaborado un análisis eminentemente descriptivo e interpretativo que nos permite sistematizar los desafíos metodológicos que puedan ser de utilidad para otras investigaciones en contextos similares de precarización institucional.

Después de una revisión bibliográfica especializada, debemos considerar dos premisas. En primer lugar, que la identificación de medios de comunicación informativos peruanos requiere de una fuente de datos formales fundamentados en la regulación local. En segundo lugar, debido a que los estudios se basan en una perspectiva analógica que categoriza a los medios con base en su soporte, consideramos que la convergencia y orientación multiplataforma del fenómeno digital pone en cuestión la manera de analizar y categorizar a los medios, dando un mayor predominio a las marcas mediáticas.

Con el objetivo de documentar la complejidad del sistema de medios peruanos, empezamos a realizar acercamientos preliminares similares que dieron pie a la constatación que argumentamos líneas arriba. Sin embargo, conforme fuimos avanzando en la recolección de datos, incorporando a las regiones más alejadas a la capital, corroboramos cómo los datos iniciales revelaban una mirada centralista y una realidad en donde la convergencia digital había cambiado la estructura de los medios de comunicación. Conforme avanzamos en la recogida de datos, optamos por mantener una mirada flexible, permitiendo que los datos levantados cuestionen nuestros protocolos metodológicos de recolección de cifras y de análisis, colocando en nuestra agenda la necesidad de triangular las fuentes de información para darle una mayor solidez a la data sistematizada.

Considerando lo planteado por la literatura metodológica, se consideró oportuno triangular fuentes estatales, públicas y auto referenciadas. Entendemos por fuentes estatales aquellas administradas por instituciones gubernamentales en ejercicio de sus funciones otorgadas por la ley. Consideramos como fuentes públicas a las iniciativas privadas que elaboran registros de medios para su mayor reconocimiento en la sociedad. Finalmente, las fuentes auto referenciadas remiten a la publicación por el medio de su identidad corporativa. La triangulación propuesta permite integrar tres voces para una mayor pluralidad y alcance: estatal, institucional y comunicacional. Se aplicó como criterio para su reconocimiento como medio informativo su registro en la fuente estatal y en alguna de las dos fuentes adicionales: privada o auto referenciada. 
Paraseleccionar las fuentes públicas se siguió el criterio de legalidad, esto es, emplear el registro en fuentes que otorguen reconocimiento de acuerdo con la ley nacional. La información sobre radio y televisión se obtuvo de bases de datos del Ministerio de Transporte y Comunicaciones (Estaciones autorizadas, n.d.). Las frecuencias de radio y de televisión son propiedad del Estado y el ente encargado por ley de asignar las frecuencias es el MTC, dándole un estatus legal y formal a quien la emplea.

En el caso de las frecuencias de radios, se obtuvieron 5745 registros y 2021 de televisión. En ambos casos no se diferenciaban tipos de frecuencia: emisora y repetidora. Por otro lado, las frecuencias se asignaban a personas jurídicas que no necesariamente coincidían con las marcas mediáticas reconocidas por las audiencias. Ante esta situación, se diseñó un protocolo de reducción de personerías jurídicas repetidas hasta obtener el número de marcas radiales. Este trabajo se desarrolló entre octubre de 2019 y enero de 2020, obteniendo un total de 995 marcas radiales y 118 marcas televisivas ${ }^{1}$ que operan formalmente en el Perú, de las cuales se tiene la certeza que 347 radios y 85 canales de televisión cuentan con espacios informativos. Se detectaron numerosas frecuencias asignadas a empresas o personas naturales de las cuales no se cuenta con información alguna de sus operaciones, las cuales no fueron consideradas en el registro.

En el caso de la prensa escrita, se optó por solicitar la información a la Superintendencia Nacional de Administración Tributaria (SUNAT)² , considerando que toda empresa periodística debe tener un registro tributario que le da un estatus formal. Es así como se obtuvo un total de 299 publicaciones de periodicidad diaria, semanal, quincenal, mensual y trimestral. Se aplicó un protocolo similar de reducción, obteniendo un total de 187 publicaciones. Las restantes fueron descartadas (112) al no poder comprobar ediciones recientes durante 2019 o información de su funcionamiento activo. Asimismo, destacamos aquellas que producían información de la actualidad (181 publicaciones). La tabla 1 muestra el resultado de la aplicación de protocolos de reducción de las bases de datos obtenidas.

1. Incluyen marcas de señal analógica, televisión digital terrestre y por pago. La televisión peruana convive hoy entre el sistema analógico y digital, además de las empresas de televisión por pago. Se tiene programado el apagón analógico para el año 2022 (Decreto Supremo $N^{\circ}$ 006-2020-MTC, 2020). La televisión gratuita digital terrestre es un fenómeno reciente impulsado desde el Estado. En la actualidad, solo se cuenta con 30 frecuencias asignadas en esa categoría (Canales que transmiten TDT, n.d.). Por otro lado, la información pública sobre las empresas de cable es limitada, más aún la grilla de marcas televisivas y la programación de los canales exclusivos.

2. A diferencia de los casos de radio y televisión, estos datos se solicitaron formalmente, invocando la Ley de Acceso a la Información Pública ( $N^{\circ} 27806$ ). 
Tipo de soporte

Marcas en general

995

118

187

1300

Total
Marcas con producción

informativa

347

85

181

Tabla 1. Marcas identificadas en bases de datos oficiales

Fuente: Elaboración propia.

La información obtenida de bases de datos públicas ofrece el panorama de las personas jurídicas y naturales detrás de la autorización legal para ejercer como medio de comunicación. Las bases de datos obtenidas de las dos fuentes oficiales (MTC y SUNAT) fueron cotejadas en una segunda etapa con varias bases de datos privadas de acceso público para completar la obtenida en las bases de datos públicas.

Para seleccionar las fuentes públicas se evaluaron plataformas que reunieran las siguientes condiciones: acceso abierto y gratuito, oferta de información regional del país, dinámicas participativas para añadir y actualizar información, y acceso para corroborar el funcionamiento del medio. En el caso de las radios, se empleó la base Radios en América Latina (https://www.radio-america-latina.org) y Planeta radios (http://www.planetaradios.com). Para la televisión, se emplearon las listas publicadas en Wikipedia (Televisión en el Perú, 2020) y Adonde.com. Otras bases de datos privadas de televisión estaban excesivamente centralizadas en las casas televisoras con sede en la capital. En el caso de prensa escrita, se emplearon las bases de datos privadas de PrensaEscrita.com, TN Relaciones (http://www. tnrelaciones.com) y Perú Quiosco (peruquiosco.pe). Finalmente, con la información disponible, se indagaron y revisaron las páginas web publicadas por los propios medios de comunicación.

El protocolo de recolección de datos estableció como punto de partida el concepto de medio informativo, "todo medio de comunicación, siempre y cuando contenga programas y secciones informativas" (Worlds of Journalism, 2012, p. 1) y la tradicional clasificación radio, televisión y prensa escrita. Luego de la extracción preliminar de las bases de datos públicas y privadas, se inició la revisión de la información publicada por los propios medios de comunicación. Para seleccionar y validar las fuentes auto referenciadas se consideraron tanto páginas web como redes sociales (Facebook, Twitter y YouTube), siendo registradas aquellas activas en los últimos seis meses al momento de la verificación. 
El protocolo incluyó el registro de la marca mediática, el alcance, el tipo de medio y si poseía programas informativos, el tipo de verificación, el número y las regiones en las que opera, y el enlace de donde se obtuvo la información. En este contexto, consideramos marca mediática al nombre con el que el medio es conocido. En segundo lugar, consideramos el alcance mediático en tres categorías: local (una región), regional (2 a 17 regiones) y nacional (18 a 25 regiones, 75\% del territorio nacional). En tercer lugar, para identificar el tipo de medio, en aquellos con una página web o redes sociales se revisó si mostraban información de su programación. En lque publicaban su programación, se codificó si poseían segmentos o programación informativos. Se registró el tipo de verificación en dos códigos, en línea cuando la información fue obtenida a través de medios digitales e in situ cuando la información fue obtenida por el traslado de un equipo de campo a la zona. Como parte de una prueba piloto, se visitó una región de frontera para verificar la información. Asimismo, se registró el número y las regiones en las que opera el medio. Sobre este aspecto, encontramos dos dificultades: medios que no publican información al respecto y la dispersión de la información. Finalmente, se registró el enlace digital para posteriores verificaciones.

\section{RESULTADOS}

Los desafíos metodológicos encontrados en la recolección de datos han sido agrupados en dos áreas: las fuentes de información necesarias para dar legitimidad a la representación de un ecosistema informativo y las categorizaciones empleadas para su organización.

\section{Fuentes de información para un ecosistema}

Sobre las fuentes de información, es necesario poner en discusión, por un lado, el peso de la formalidad y la legislación de los medios de comunicación y, por otro, la preponderancia de la huella digital. Frente al tema de la formalidad y regulación de los medios, es probable que el contexto sociocultural contribuya a una crónica debilidad institucional. Es así que nos enfrentamos al dilema de instituciones formales con prácticas informales. Un medio de comunicación formal se ajusta a la normativa y regulación. No obstante, encontramos que dentro de la formalidad existen formas de operación informales.

El caso de las radios nos ofrece una interesante ilustración. Bethel Radio (http:// bethelradio.fm/) es un medio radial orientado al culto religioso, de alcance nacional, con un noticiero que se emite dos veces al día. De todas las frecuencias identificadas con esta radio, la mayor parte está asignada a la razón social Asociación Cultural Bethel, tal como consta en los registros del MTC. Un menor número de frecuencias están asignadas a personas naturales o asociaciones con otra nominación. 
Este caso ilustra la figura de una radio formal mediante el indicador de la razón social a la cual están adscritas las frecuencias. Sin embargo, tenemos otros casos de medios informativos que operan subarrendando frecuencias, como estrategia para tener un mayor alcance. Un ejemplo de ello es la radio regional La Ribereña (http:// gruporiberena.com/), con un segmento informativo con alcance a 12 regiones del país. Se encontraron hasta 20 razones sociales a las cuales están adscritas sus frecuencias. Otro caso similar es Radio María (https://www.radiomariaperu. org/), de corte religioso y alcance nacional, con un programa informativo, con frecuencias adscritas a 12 razones sociales diferentes. El fenómeno del subarriendo de frecuencias no es un acto ilegal, si bien no queda clara la responsabilidad de los diferentes actores involucrados con respecto a los contenidos y las condiciones laborales de los que producen la información.

La situación es más estable en el caso de la televisión, que presenta congruencia entre las razones sociales y las marcas mediáticas. En el caso de la prensa, existen algunas regulaciones nacionales a ser tomadas en cuenta. El depósito legal obliga a autores y editores a entregar un número de ejemplares a la Biblioteca Nacional del Perú (BNP), para preservar el acervo bibliográfico nacional (Depósito legal, n.d.). De 181 publicaciones periódicas informativas, en 49 casos no se pudo verificar el registro del depósito legal a través de la base de datos de la BNP. No podemos identificar si se debe a problemas de registro de la base de datos pública o a omisiones de las empresas periodísticas. Otro caso que podemos mencionar es Don Jaque, un diario de circulación local en la región Madre de Dios, que se define como un diario judicial. Cuenta con registro tributario, mas no se identificó el depósito legal en la BNP. En otros siete diarios no encontramos indicio del registro del depósito legal, tanto en las versiones impresas digitalizadas o la BNP. Esta situación se repite en los diarios locales Pregón (Apurímac), Hocicón (Ayacucho), El Cumbe (Cajamarca), El Siglo (Huánuco), Nuevo Norte (La Libertad), y el diario Hoy (San Martín). Sin embargo, todos los medios mencionados tienen una razón social única e identificable, lo que podría deberse a la necesidad de registrarse como negocio para obtener ingresos por publicidad.

Sobre la huella digital de los medios, esto nos puso ante la brecha digital entre los centros urbanos de mayor densidad poblacional, con mayor conectividad, y los centros urbanos y rurales de menor densidad poblacional y con menor conectividad. De las 347 radios informativas o con segmentos informativos, todas tenían huella digital. Por lo tanto, la brecha digital entendida como acceso o no acceso a recursos digitales es cuestionable. Sin embargo, hay diferencias en la calidad de la huella digital tal como lo ilustramos a continuación.

Algunas radios cuentan con una página web que brinda información de la radio, en especial de su programación. Un ejemplo de alcance local al sur del Perú 
es la radio Contacto Sur (http://radiocontactosur.com/programacion/), cuya página web muestra una programación variada y varios segmentos informativos. En este caso, la plataforma es un soporte de emisión de la radio analógica. Sin embargo, no todas las marcas radiales identificadas cuentan con este nivel de presencia digital. De 347 radios informativas con huella digital, 129 tenían como principal soporte digital una cuenta de Facebook. Algunas cuentas eran más activas que otras. Un ejemplo de cuenta activa es la de Radio Sepahua (Radio Sepahua 100.5 FM - La señal que integra, n.d.), de alcance local (Región Loreto) y con programación variada, con 4025 seguidores (21/2/2020). La cuenta de Facebook publica entrevistas de la emisora, noticias locales y de regiones cercanas. Un caso opuesto es la cuenta de Facebook de Radio Amistad 99.1 FM (Radio Amistad 99.1 FM - Más fuerte que nunca, n.d.) de alcance local (Región Cajamarca), con programación variada (342 seguidores al 12/8/2021). La última publicación data del 22 septiembre de 2017. Más que la accesibilidad a los recursos digitales, nos encontramos con competencias digitales diferentes que hacen más visible a la emisora radial.

Con respecto a la televisión, de 85 empresas con producción informativa, encontramos que todas poseen alguna forma de huella digital. La mitad cuentan con páginas web y el resto emplea principalmente una cuenta de Facebook para su difusión. Podemos establecer tres tipos de presencia digital en la televisión: (1) las páginas web cuya función es soporte de emisiones en vivo de los programas, además de información relativa a la casa televisora, (2) cuentas de Facebook en donde publican emisiones de los programas de televisión, y (3) páginas web que publican información sobre la televisora, algunas incluyen la programación, pero no publican los contenidos.

Un caso que ilustra el primer tipo es la televisora de orientación religiosa JN19 (http://www.jn19television.com/), con sede en Lima, con espacios informativos y alcance nacional. Su página web ofrece la emisión analógica en vivo. Una situación similar, pero a través de Facebook, es la de la televisora local Canal 33 de la región Lambayeque. No ofrece mucha información del canal, pero en una cuenta Facebook los periodistas de Pulso TV Canal 33 (Pulso TV Canal 1, n.d.) publican las emisiones grabadas de sus programas informativos. El tercer tipo se ilustra con TV SUR (https://www.tvsur.com.pe/), con sede en Puno. Posee una programación variada, con segmentos informativos. Su página web muestra los programas que produce y su programación. Personal de la televisora produce la información noticiosa de la web.

En el caso de la prensa informativa, de 181 diarios todos contaban con referencia digital, la cual varía significativamente. Podemos establecer tres tipos de presencia digital: (1) las páginas web que son versiones digitales del diario impreso, 
(2) plataformas de suscripción cuya función es comercializar la versión digitalizada del diario impreso, y (3) la cuenta de Facebook que promueve el consumo de la venta de la versión impresa.

El primer tipo es una página web que eventualmente publica las mismas noticias de la versión impresa y ofrece el servicio de actualización de las noticias con detalles de los acontecimientos durante el día. Un ejemplo es el diario regional La Industria (http://www.laindustria.pe/), con sede en la región La Libertad. Este cuenta con dos versiones regionales (Ancash, http://www.laindustria.pe/ ancash y Lambayeque, https://www.laindustriadechiclayo.pe/). Cada versión regional tiene una página web que publica información actualizada de la versión impresa y de la portada de la versión impresa. En la web confluye lo publicado en las redes sociales.

Un segundo tipo de presencia digital son las plataformas de pago para obtener las versiones impresas digitalizadas, es decir el diario impreso de papel en archivo PDF. Es así como tenemos el diario Correo (https://diariocorreo.pe/), de alcance regional con sede en Lima, con 13 versiones regionales. Su página web publica versiones actualizadas de las noticias de la versión impresa. Las versiones regionales no poseen una página web autónoma, sus publicaciones están contenidas en la web central y etiquetadas por ubicación geográfica. Adicionalmente, la versión impresa digitalizada es comercializada a través de la plataforma Perú Quiosco (2020) que muestra las portadas de los diarios. Finalmente, como tercer tipo tenemos las cuentas de Facebook. Ese es el caso del Diario El Clarín de Amazonas (Diario El Clarín Amazonas, n.d.), de alcance local. Emplea su cuenta de Facebook para publicar las portadas de su edición diaria, no los contenidos, como estrategia para impulsar la compra del diario impreso.

\section{Categorizaciones}

Otro desafío metodológico en torno a sistematizar los medios informativos está relacionado con las categorizaciones empleadas para organizar el sistema mediático y la jerarquía organizativa de las empresas mediáticas.

Sobre el primero, tradicionalmente se han categorizado a los medios de comunicación por su soporte: radio, televisión y prensa. En el caso peruano, el Ministerio de Transporte y Comunicación gestiona las frecuencias de radio y televisión. La prensa, al ser un producto editorial, es gestionada a través de la Biblioteca Nacional del Perú, perteneciente al Ministerio de Educación. En el actual ecosistema mediático, la categorización radio, televisión y prensa se enfrenta al desafío de la convergencia digital. La categorización marcas en las que convergen varios soportes -radial, televisivo o impreso- ocasiona en algunos casos un triple o doble registro. 
Podemos examinar el caso de Exitosa, de alcance nacional, con Exitosa Radio, Exitosa TV y Exitosa Diario (https://exitosanoticias.pe/v1/). La señal de televisión es una emisión en vivo de la cabina de radio. Las noticias publicadas en la versión impresa del diario son reproducidas a través de su página web. Nos preguntamos ante esta situación si debe ser considerado como un medio o como tres medios de comunicación, como una marca o como tres marcas mediáticas. Esto con la finalidad de evitar la sobre o sub representación de un medio de comunicación.

¿Qué criterios se pueden emplear para configurar una unidad de observación? A primera vista, podría considerarse el soporte más reconocido para un medio de comunicación. Esto significa que, si un medio es históricamente reconocido como una radio, debería ser registrado solamente como un medio radial e ignorar sus otros soportes. No obstante, la evolución de los medios de comunicación que potencian la función informativa en nuevas plataformas pone en duda la conveniencia de este. Un segundo criterio es si la programación informativa es equivalente entre un soporte y otro, para considerarlo como un solo medio. Esto es posible en el caso de radio y televisión que presentan la misma programación, pero no sucede en el caso de televisión y prensa escrita, cuya organización del contenido es diferente. Otro criterio son los equipos periodísticos del medio. Si existen equipos periodísticos autónomos en un medio y otro podrían ser considerados como medios diferentes. Si un solo equipo periodístico produce información para tres soportes, podría ser considerado un solo medio.

Un caso de análisis es Radio Programas del Perú (https://rpp.pe/), históricamente conocido por la frase la radio está más cerca de la gente. De alcance nacional, su programación es fundamentalmente informativa. Además de RPP radio, también tenemos RPP TV. En este caso, la programación de ambos soportes coincide con algunos programas o segmentos informativos y en otros, la programación es diferente.

Sobre el segundo desafío metodológico, relacionado con la jerarquía organizativa de las empresas mediáticas, nos enfrenta a conceptos como grupo o conglomerado mediático, medio de comunicación y marca mediática. Por conglomerado entendemos una entidad organizativa que agrupa a varios medios de comunicación o marcas mediáticas. Una segunda categoría conceptual es el medio de comunicación, que puede ser una organización empresarial, con o sin fines de lucro. La marca mediática es la denominación con la que un medio de comunicación es conocido ante su público.

Los grupos empresariales mediáticos adquieren importancia, en la medida en que nos plantea el dilema de la concentración de medios y la injerencia de los intereses del conglomerado mediático en los periodistas que producen la información. Un ejemplo de ello es el Grupo ATV que administra seis canales de televisión, como ATV, ATV+, ATV Sur, La Tele, América Next y ATV.pe (medio digital). 


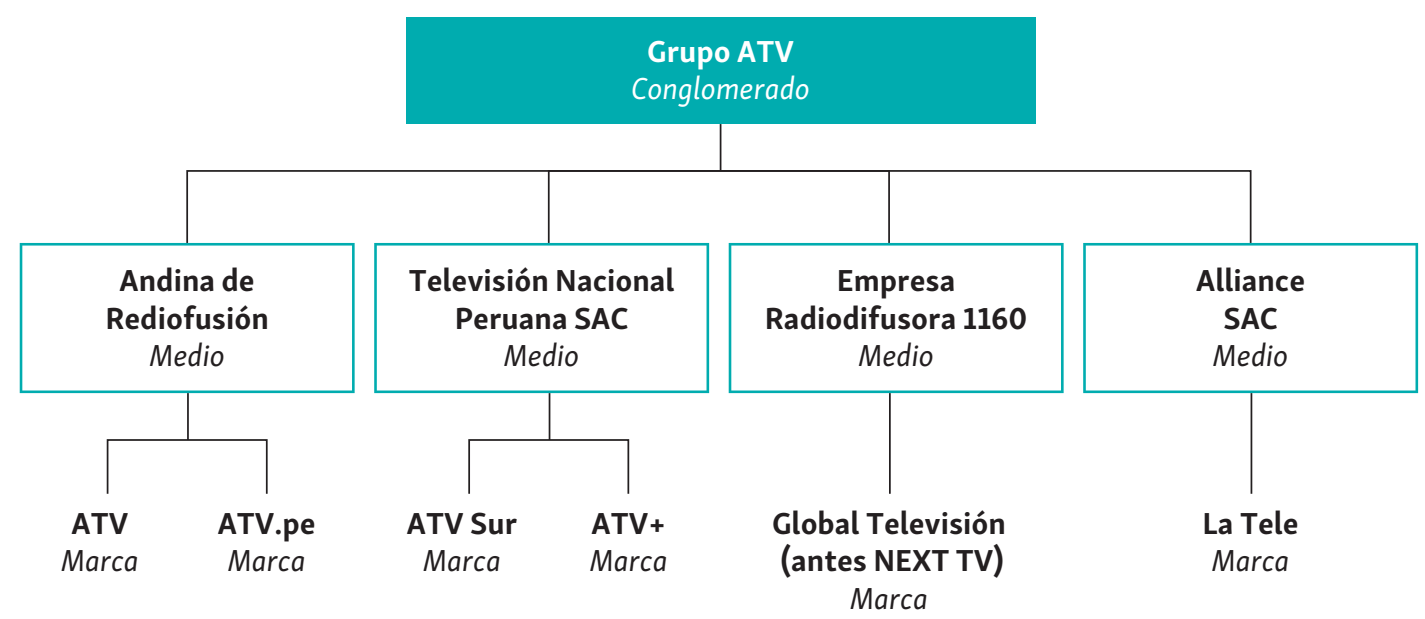

Figura 1. Medios y marcas involucrados en el conglomerado Grupo ATV

Fuente: Elaboración propia.

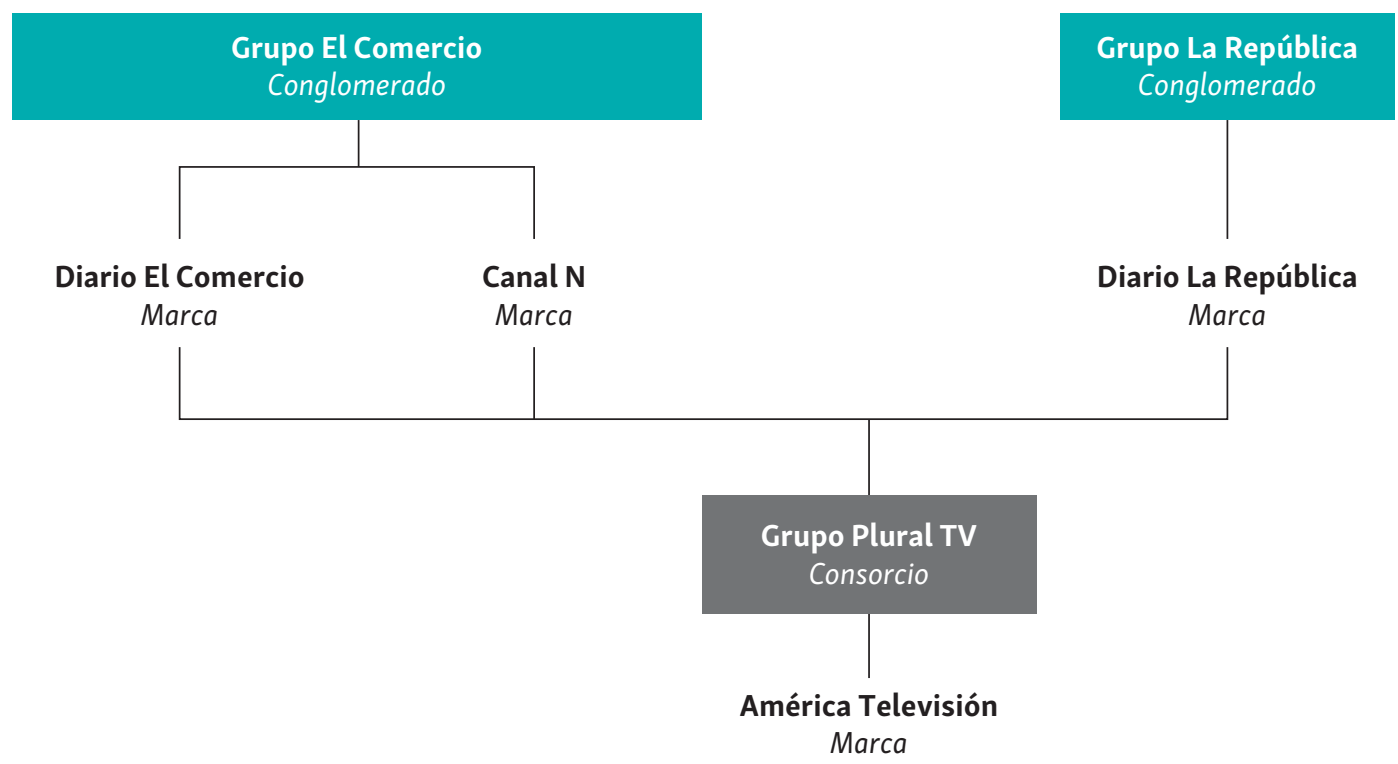

Figura 2. Estructura de la propiedad de América Televisión

Fuente: Elaboración propia.

No obstante, la jerarquía entre conglomerado y medios de comunicación dependientes no se aplica a todos los casos y resulta más compleja. Es el caso del medio América Televisión, la cual opera bajo la razón social Corporación Peruana de Radiodifusión, propiedad del Grupo Plural TV. Este se define como un consorcio, conformado por marcas como el diario El Comercio y Canal $N$ (propiedad del grupo El Comercio), el diario La República (propiedad del grupo La República), y Next TV, propiedad del Grupo Plural TV en alianza con el Grupo ATV. Estos medios, al constituir el consorcio, se vuelven propietarios de América Televisión. 
Este caso abrió el debate en torno a la concentración de la propiedad de medios informativos en el Perú, por la influencia del Grupo El Comercio, que además de tener injerencia sobre uno de los canales de televisión más reconocidos en el país (América Televisión), es propietaria de los diarios El Comercio, El Trome, Perú 21 y Gestión; el canal de televisión Canal N, y recientemente adquirió la mayoría de las acciones del Grupo Epensa, que administraba los diarios Correo, Ojo, Ajá y El Bocón ${ }^{3}$.

\section{DISCUSIÓN}

El objetivo de este artículo ha sido sistematizar los desafíos metodológicos encontrados al cartografiar los medios informativos en el Perú. Las fuentes de información para representar el ecosistema mediático resultaron un aspecto importante en la discusión metodológica, destacando el aspecto de la formalidad y la regulación de los medios de comunicación. Resulta insuficiente considerar la formalidad e informalidad como dinámicas excluyentes entre sí. Es necesario profundizar en el fenómeno de medios formales con prácticas informales, su repercusión en las condiciones laborales de los periodistas en el caso de los medios informativos y la responsabilidad del medio sobre los contenidos producidos. Si bien Zelizer (2013) señala el sesgo del estudio de un mundo periodístico muy estrecho, también es probable que la etiqueta de periodismo democrático haya supuesto que esta no alberga prácticas informales que condicionan el trabajo de los periodistas.

Adicionalmente, nuestro estudio encuentra diferencias en la calidad de la huella digital, que remite a competencias digitales que articulan distintos tipos de presencia digital. Es por ello que se hace importante incluir criterios de subcodificación, que distingan distintas formas de presencia digital.

Las categorizaciones son parte fundamental en la representación de un ecosistema mediático. La organización política de los medios de comunicación impone la categorización a través de su soporte. Sumado al fenómeno de convergencia digital, puede producir distorsiones en la representación de un sistema de medios, creando la ilusión de más medios de los que realmente existen. Se hace necesario concordar en criterios para el registro de medios, que consideren la naturaleza multimedial del sistema. En ese sentido, no solo se requiere tomar en cuenta nuevas

3. En 2013, el grupo El Comercio adquirió 54\% de las acciones de EPENSA y ABS, obteniendo control de $80 \%$ de los diarios impresos. En noviembre del mismo año se interpuso una demanda por afectación a la libertad de expresión y pluralismo informativo. En 2021 se declaró fundada la demanda y solicita declarar nulo el contrato de compraventa. Actualmente el grupo empresarial apelará el fallo. 
formas de ejercer la profesión (Schmidt, 2008, 2010), sino también nuevas formas de categorizar y comprender la naturaleza del medio. El universalismo teórico tan impregnado en el rol del periodista (Hanitzsch et al., 2019) también es aplicable a la consideración del medio y su soporte, en la línea de las reflexiones planteadas por Waisbord (2009). De manera tentativa se podrían formular grados de medialidad: alta, moderada y baja. A cada una de ellas desarrollar indicadores en donde se integre la preponderancia del soporte. Por ejemplo, en el caso de la radio, su sintonía o el rating, en televisión. En el caso de las plataformas tecnológicas, se puede considerar su tráfico y alcance. No queda claro la integración de la participación publicitaria y el alcance, sobre todo con relación a los soportes digitales.

En esa misma línea, las dinámicas empresariales de los medios de comunicación conllevan una revisión de las categorías relacionadas con la jerarquía organizativa de los medios. Se hace necesario distinguir entre la figura del conglomerado o grupo mediático, el medio de comunicación como empresa y la marca mediática con la que el público reconoce al medio, y observarlas de manera flexible. Esto se encuentra en la línea señalada por Hanitzsh y sus colegas (2019) del periodismo como institución en recreación discursiva, no solo aplicado a la producción de noticias sino también a las dinámicas empresariales que las rodean. A nivel metodológico, se hace necesario profundizar y tipificar la articulación de dichas categorías conceptuales de la organización mediática, producto de las dinámicas empresariales.

A modo de investigaciones futuras, hace falta comprender las motivaciones detrás de las formas informales dentro de los medios de comunicación y su repercusión en las condiciones del ejercicio periodístico. Asimismo, profundizar en las diferentes competencias digitales, la valoración por parte del medio y el impacto que la presencia digital tiene en los medios de comunicación. Queda pendiente visibilizar los datos recogidos y generar una dinámica colaborativa y participativa que afine la información recogida para este estudio.

\section{FINANCIAMIENTO}

Título del proyecto: Cartografía de medios informativos peruanos

Años de financiamiento: 2019-2020

Institución: Instituto de Investigación Científica de la Universidad de Lima

País: Perú 


\section{REFERENCIAS}

Acevedo, J. (2016). Perú. Elecciones, medios de comunicación y redes sociales en el Perú (2016) (Peru. Elections, media, and social networks in Peru (2016)). The Alliance for Networking Visual Culture. Retrieved from https://scalar.usc.edu/works/medios-y-elecciones/per

Acevedo, J. (2017). Los medios de comunicación como actores políticos. Cobertura de las elecciones presidenciales de 2016 en el Perú (The media as political actors. Coverage of the 2016 presidential elections in Peru). In D. Bruzone \& M. Papaleo (Coords.), Comunicación para la resistencia. Conceptos, tensiones y estrategias en el campo político de los medios (Communication for resistance. Concepts, tensions and strategies in the political field of the media) (pp. 99-126). CLACSO. Retrieved from http://biblioteca.clacso.edu.ar/clacso/gt/20181221054453/Comunicacion-para-laresistencia.pdf

Becerra, M. \& Mastrini, G. (2006). Periodistas y magnates. Estructura y concentración de las industrias culturales en América Latina (Journalists and tycoons. Structure and concentration of cultural industries in Latin America). Prometeo.

Becerra, M. \& Mastrini, G. (2009). Los dueños de la palabra. Acceso, estructura y concentración de los medios en la América Latina del Siglo XXI (The owners of the word. Access, structure, and concentration of the media in the Latin America of the 21st century). Prometeo.

Becerra, M. \& Mastrini, G. (2017). La concentración infocomunicacional en América Latina (2000-2015): Nuevos medios y tecnologías, menos actores (The infocommunication concentration in Latin America (2000-2015): New media and technologies, fewer actors). Universidad Nacional de Quilmes.

Boczkowski, P. J. \& Mitchelstein, E. (2019). The politics of contextualization in the contextualization of political communication research. Political Communication, 36(4), 676-679. https://doi.org/10.1080/10584609.2019.1670903

Canales que transmiten TDT (Channels that broadcast DTT). (n.d.) Televisión Digital Terrestre. Retrieved from http://www.tdt.pe/File/detalle_canales.pdf

Castilla, O., Castro, J., \& Yañez, L. (2016). Dueños de la noticia. Ojo Público, Lima. https://duenosdelanoticia.ojo-publico.com/articulo/los-duenos-de-la-noticia/

Chanduvi, E. (2014). Media concentration threatens press freedom in Peru. Latin American and Iberian Institute. Retrieved from https://digitalrepository.unm.edu/cgi/viewcontent. cgi? article $=15233 \&$ context $=$ notisur

Decreto Supremo n 006-2020-MTC. Decreto Supremo que modifica el numeral 15.1 del artículo 15 y el artículo 17 del Plan Maestro para la Implementación de la Televisión Digital Terrestre en el Perú, aprobado por Decreto Supremo No 017- 2010-MTC (Supreme Decree No. 006-2020-MTC. Supreme Decree that modifies numeral 15.1 of article 15 and article 17 of the Master Plan for the Implementation of Digital Terrestrial Television in Peru, approved by Supreme Decree No. 017-2010-MTC). (2020). Retrieved from https://cdn.www.gob.pe/uploads/document/file/514529/DS_006-2020-MTC.pdf

Depósito legal. (n.d.). Biblioteca Nacional del Perú. Retrieved from https://www.bnp.gob.pe/servicios/deposito-legal/

Diario El Clarín Amazonas. (n.d.). Home (Facebook page). Retrieved from https://www.facebook.com/ElClarinAmazonas.pe/ 
Estaciones autorizadas (Authorized stations). (n.d.). Ministerio de Transportes y Comunicaciones. Retrieved from https://portal.mtc.gob.pe/comunicaciones/autorizaciones/radiodifusion/ estaciones_autorizadas.html

Freundt-Thurne, Ú., Pita, C., \& Ampuero, M. J. (2013).Mapping Digital Media: Peru. Open Society Foundations. Retrieved from https://www.opensocietyfoundations.org/publications/ mapping-digital-media-peru\#publications_download

Gargurevich, J. (2012). Los medios masivos de información en el Perú, 1980-2012 (Mass media in Peru, 1980-2012). Conexión, 1(1) 11-31. Retrieved from revistas.pucp.edu.pe/index.php/ conexion/article/download/11555/12079

Golding, P. (1977). Media professionalism in the Third World: The transfer of an ideology. In J. Curran, M. Garevitch, \& J. Woollacott (Eds.), Mass communication and Society (pp. 291-308). Edward Arnold.

Gurevitch, M. \& Blumler, J. G. (2004). State of the Art of Comparative Political Communication Research: Poised for Maturity. In F. Esser \& B. Pfetsch (Eds.), Comparing political communication: Theories, cases, and challenges (pp. 325-343). : Cambridge University Press. https://doi.org/10.1017/CB09780511606991

Hanitzsch, T., Hanusch, F., Mellado, C., Anikina, M., Berganza, R., Cangoz, I.,... \& Virginia-Moreira, S. (2011). Mapping journalism cultures across nations: A comparative study of 18 countries. Journalism Studies, 12 (3), 273-293.

https://doi.org/10.1080/1461670X.2010.512502

Hanitzsch, T., Hanusch, F., Ramaprasad, J., \& De Beer, A. (Eds.). (2019). Worlds of Journalism: Journalistic Cultures Around the Globe. New York, NY: Columbia University Press. https://doi.org/10.7312/hani18642

Hanitzsch, T. \& Vos, T. (2017). Journalistic Roles and the Struggle Over Institutional Identity: The Discursive Constitution of Journalism: Journalistic Roles and Institutional Identity. Communication Theory, 27(2), 115-135. https://doi.org/10.1111/comt.12112

Hanitzsch, T. \& Vos, T. (2018). Journalism beyond democracy: A new look into journalistic roles in political and everyday life. Journalism, 19(2), 146-164.

https://doi.org/10.1177/1464884916673386

Impresos (Printed media). (n.d.). Ojo Público. Retrieved from http://peru.mom-rsf.org/es/medios/prensa/

Lainez, Z. (2014). Peru: the media tread carefully. British Journalism Review, 25(4), 56-60. https://doi.org/10.1177/0956474814562774

Lovatón, M. D. (2016). Estándares interamericanos sobre pluralidad de información y concentración de medios (Inter-American standards on plurality of information and media concentration). In ¿Puede acapararse la libertad? Libertad de expresión y concentración de medios en el Perú (Can freedom be hoarded? Freedom of expression and media concentration in Peru) (pp. 95-139). Pontificia Universidad Católica del Perú.

Masterton, M. (1996). Asian values in journalism. Singapore: Asian Media Information and Communication Centre. 
Mellado, C. (2009). Periodismo en Latinoamérica: Revisión histórica y propuesta de un modelo de análisis (Latin American journalism: A review of five decades and a proposal for a model of analysis). Comunicar, 33, 193-201. https://doi.org/10.3916/c33-2009-01-011

Nerone, J. (2013). The historical roots of the normative model of journalism. Journalism, 14(4), 446-458. https://doi.org/10.1177/1464884912464177

Norris, P. (Ed.). (2009). Public Sentinel: News media and governance reform. The World Bank.

Open Society Foundations. (2010, October 4). Investigative journalists take on Peruvian corruption (Press release). Retrieved from https://www.opensocietyfoundations.org/ press-releases/investigative-journalists-take-peruvian-corruption

Pintak, L. (2014). Islam, identity and professional values: A study of journalists in three Muslimmajority regions. Journalism, 15(4), 482-503. https://doi.org/10.1177/1464884913490269

Protzel, J. (2014). Media Systems and Political Action in Peru. In M. A. Guerrero \& M. Márquez-Ramírez (Eds.), Media Systems and Communication Policies in Latin America (pp. 82-99). Palgrave Macmillan. https://doi.org/10.1057/9781137409058

Protzel, J. (1994). El paradigma del Príncipe: el líder, la Razón de Estado y los medios electrónicos (The Prince paradigm: the leader, the Reason of State, and the electronic media). Contratexto, 7, 21-29. https://doi.org/10.26439/contratexto1994.n007.1951

Pulso TV Canal 1. (n.d.). Home (Facebook page). Retrieved from www.facebook.com/Pulso-TV-Canal-33-1135222749822542

Radio Amistad 99.1 FM - Más fuerte que nunca. (n.d.) Home (Facebook page). Retrieved from https://www.facebook.com/TuRadioAmistadFM

Radio Sepahua 100.5 FM - La señal que integra. (n.d.). Home (Facebook page). Retrieved from https://www.facebook.com/radiosepahua

Santillán, J. (2017). Acerca de la concentración de medios (About media concentration). Cultura, 31, 141-156. https://doi.org/10.24265/cultura.2017.v31.07

Schmidt, V. A. (2008). Discursive Institutionalism: The Explanatory Power of Ideas and Discourse. Annual Review of Political Science, 11, 303-326. https://doi.org/10.1146/annurev.polisci.11.060606.135342

Schmidt, V. A. (2010). Taking ideas and discourse seriously: explaining change through discursive institutionalism as the fourth "new institutionalism". European Political Science Review: EPSR, 2(1), 1-25. https://doi.org/10.1017/S175577390999021X

Sinclair, J. (1999). Latin American television: A global view. Oxford University Press.

Televisión en Perú (Television in Peru). (2020, February 8). Wikipedia. Retrieved from https://es.wikipedia.org/w/index.php?title=Televisi\%C3\%B3n_en_Per\%C3\%BA\&oldid=123573703

Vivas, F. (2008). En vivo y en directo. Una historia de la televisión peruana (Live. A history of Peruvian televisión). Lima, Peru: Universidad de Lima.

Waisbord, S. (2000). Watchdog journalism in South America: News, accountability, and democracy. Columbia University Press.

Waisbord, S. (2009). Research directions for global journalism studies. Ideas from Latin America. Journalism, 10(3), 393-395. https://doi.org/10.1177/1464884909102586 
Waisbord, S. \& Mellado, C. (2014). De-westernizing communication studies: A reassessment. Communication Theory, 24(4), 361-372. https://doi.org/10.1111/comt.12044

Worlds of Journalism. (2012). Field Manual (PDF). Retrieved from https://worldsofjournalism.org/methodological-documentation/

Xu, X. (2005). Demystifying Asian values in journalism. Marshall Cavendish.

Zelizer, B. (2004). Taking journalism seriously: News and the academy. Sage.

Zelizer, B. (2013). On the shelf life of democracy in journalism scholarship. Journalism, 14(4), 459-473. https://doi.org/10.1177/1464884912464179

\section{SOBRE LAS AUTORAS}

JESSICA RETIS, docente. Full Professor. Directora de la Maestría en Periodismo Bilingüe en la Universidad de Arizona. Licenciada en Comunicaciones (Universidad de Lima). Maestra en Estudios Latinoamericanos (UNAM). Doctora en América Latina Contemporánea (Universidad Complutense de Madrid). Áreas de investigación: migración internacional, Latinoamérica, diásporas, comunidades transnacionales, estudios de periodismo bilingüe, medios latinos en Europa, Norteamérica y Asia. Coautora de Latin Americans in London: Narratives of Migration, Relocation and Belonging (Palgrave). Coeditora de The Handbook of Diasporas, Media and Culture (Willey).

iD https://orcid.org/0000-0003-0665-9837

LILIAN KANASHIRO, docente de la Facultad de Comunicación de la Universidad de Lima. Licenciada en Comunicaciones (Universidad de Lima). Editora de la Revista Contratexto. Magistra en Ciencias Políticas y Gobierno (Pontificia Universidad Católica del Perú). Doctoranda en Semiótica y Comunicaciones (Pontifícia Universidade Católica de São Paulo). Áreas de investigación: periodismo, sociosemiótica, comunicación política. Autora Debates presidenciales televisados en el Perú (Universidad de Lima). Coautora de El Perú y sus discursos (Pontificia Universidad Católica del Perú).

(iD) https://orcid.org/0000-0003-0486-3105

WENDY DOMENACK, docente de la Facultad de Comunicación de la Universidad de Lima. Licenciada en Comunicaciones (Universidad de Lima). Magister en Dirección de Comunicación Empresarial (Universidad Peruana de Ciencias Aplicadas). Especialización en Periodismo de Agencias (Agencia de noticias EFE y Universidad Rey Juan Carlos). Doctoranda en Sociología (Universidad Nacional Mayor de San Marcos). Áreas de investigación: periodismo, comunicación organizacional. Consultora en comunicación estratégica para el sector público y empresarial.

iD https://orcid.org/0000-0003-2575-5584 